Universidad Nacional de La Plata.

Facultad de Humanidades y Ciencias de la Educación.

Centro de Investigaciones Socio Históricas

\title{
"Cada día más empresarios": asociaciones de productores, agronegocio y estrategias institucionales en la pampa cordobesa (1995-2002)
}

\author{
"More businessmen each day": producers' associations, \\ agrobusiness and institutional strategies in Córdoba (1995-2002)
}

\begin{abstract}
Gabriel Fernando Carini *
* Centro de Investigaciones Históricas de la Universidad Nacional de Río Cuarto (CIHUNRC), Centro de Investigaciones de la Facultad de Filosofía y Humanas de la Universidad Nacional de Córdoba (CIFFyH-UNC), Argentina | gcarini@hum.unrc.edu.ar/ gabrielcarini@hotmail.com
\end{abstract}

PALABRAS CLAVE

Asociaciones de productores

Agronegocio

Estrategias institucionales

Córdoba (1995-2002)

KEYWORDS

Agrarian associations

Agrobusiness

Strategies

Córdoba (1995-2002)

\section{RESUMEN}

El objetivo del presente artículo es describir las representaciones por medio de las cuales las asociaciones de productores agrarios construyeron -discursivamente- una legitimación sobre diferentes prácticas agronómicas vinculadas a la vigencia del agronegocio. A partir de esto, se intenta explorar los desplazamientos que se operaron en los perfiles de las asociaciones tradicionales de representación de intereses agrarios a partir de la incorporación de actores que encarnaron una nueva institucionalidad centrada en aspectos técnicos antes que reivindicativos. Con ese trasfondo, tomaremos como objeto de análisis las intervenciones de la dirigencia agraria cordobesa mediatizadas en la prensa escrita. El estudio se justifica porque es un vehículo habitual por medio del cual se canalizan no solo las problemáticas sectoriales más relevantes sino también como un espacio donde se reconfiguran e interpretan un conjunto de discursos, prácticas y estrategias institucionales. Esto nos permitirá reconocer cómo los imperativos del agronegocio fueron reactualizados por los productores agrarios y asumieron sentidos diversos en el seno de sus asociaciones.

\section{ABSTRACT}

The aim of this article is to describe the representations through which agricultural producers' associations built - discursively - a legitimization of different agronomic practices related to the life of agribusiness. It tries to explore the tensions that operated in the structure of representation of agricultural interests since the incorporation of actors who embodied a new institutional framework focused on technical aspects rather than vindictive. With that background, we will take as an object of analysis interventions of Cordoba agrarian leadership mediated in the press. The study of it is a common vehicle by which the most relevant sectoral issues are channeled not only but also as a space where rewire and interpret a set of discourses, practices and institutional strategies. This will allow us to recognize how agribusiness imperatives were actualized by agricultural producers and assumed various ways within their associations. 


\section{Introducción}

La configuración de un nuevo paradigma productivo colocó en el centro de la escena a entidades agrarias centradas en cuestiones no reivindicativas, 1 muchas de las cuales habían sido creadas en el contexto de la modernización agrícola de la década de 1960. Esto planteó la emergencia de una nueva institucionalidad, especialmente en la región pampeana, de carácter innovador, dotada de un perfil diferencial respecto al de las asociaciones tradicionales -como Sociedad Rural Argentina (SRA), Federación Agraria Argentina (FAA) o Confederaciones Rurales Argentinas (CRA)- que promovió novedosas maneras de operar colectivamente dentro del aparato productivo, mediante asociaciones organizadas en torno a modernas tecnologías, rubros específicos de producción y, eventualmente, nuevos modelos productivos (Flood, 2005; Lattuada, 2006; Gras y Hernández, 2009 y 2013). Estas entidades se caracterizaron por poseer estilos de liderazgos con un alto grado de profesionalidad y con capacidad de gestión frente a los cambios que se producían, cuestión que resultó funcional a los desarrollos innovativos (Bocchicchio y Cattáneo, 2005; Alapin, 2008; Gras y Hernández, 2013). Asimismo, estas entidades introdujeron un nuevo lenguaje y enfoques diferentes para definir el papel del sector agropecuario en la economía nacional, que puede observarse mediatizado en un 'discurso tecnologizante' centrado en la consideración de que el avance tecnológico es el elemento vital para el agro, realizando una apelación al productor para que asuma una 'mentalidad empresarial', centrada en el conocimiento (Balsa, 2007, pp. 153-154).

La presencia de estas entidades en el mapa asociativo del agro nacional planteó visiones diversas sobre su incidencia en las lógicas institucionales de las entidades tradicionales o de tipo reivindicativo y la situación

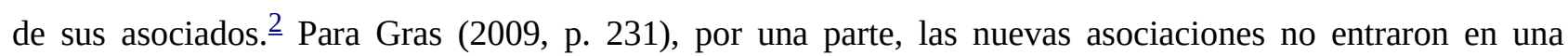
competencia por las bases sociales puesto que podría pensarse -según la autora- en una suerte de 'división del trabajo’ que implicaría para las primeras 'concentrarse’ en un ámbito específico, el de lo técnico, reforzando así su lugar de entidad especializada e impulsando a sus asociados integrarse a las de tipo 'gremial' que son las que se concentran en la 'defensa' del sector ante -por ejemplo- los efectos de las políticas económicas. Por otra parte, para Lattuada (2003 y 2006), estas nuevas asociaciones habrían llevado a replantear la estructura interna y las estrategias de las asociaciones reivindicativas -como por ejemplo, incorporando una mayor oferta de servicios sumados a su tradicional función gremial- para 'ganar posiciones’ frente a otras entidades que ‘disputan’ entre sí por las mismas bases sociales.

Con ese trasfondo, el objetivo del presente artículo es observar cómo una asociación tradicional, es decir, aquella que se caracterizaba por la función primordial la defensa, representación y reivindicación de los intereses de sus asociados elaboró -a partir de una transformación en las modalidades de interpelación de sus bases sociales- un nuevo horizonte discursivo tendiente a justificar saberes y prácticas vinculados al modelo productivo en ciernes, el agronegocio. Puntualmente, nos interesa indagar los discursos que estructuraron las asociaciones de productores tradicionales a los fines de facilitar la apropiación de las lógicas involucradas en el agronegocio y las tensiones o desplazamientos que en ese proceso se operaron en las formas históricas de comunicación con sus asociados. Para ello tomaremos como objeto de análisis la discursividad mediatizada en la prensa generada por la dirigencia rural de la Sociedad Rural de Río Cuarto (SRRC). $\underline{3}$

Partimos de la idea de que en el contexto de predominio del agronegocio, la SRRC elaboró, a partir de las intervenciones públicas de su dirigencia en la prensa, un nuevo sentido sobre lo que consideraba las modalidades más adecuadas de abordar los procesos productivos. Los lugares comunes de ese nuevo discurso fueron reactualizados por los dirigentes riocuartenses y proporcionaron su propia justificación del agronegocio. La incorporación de enunciados, términos y frases de tono tecnologizante se sumó -de forma más o menos conflictiva- a la matriz discursiva liberal-conservadora que históricamente había sostenido la entidad. Argumentamos que estas intervenciones de los dirigentes ruralistas conformaron un modo peculiar de adecuar sus discursos a los 'nuevos tiempos', construyendo explicaciones plausibles de las 
transformaciones estructurales que debieron afrontar sus bases sociales durante la década de 1990. A su vez, el empleo de estos tópicos tecnologizantes constituía un llamado a sus asociados para que profesionalizaran sus prácticas agropecuarias, asumiendo pautas de comportamiento ligadas a una racionalidad empresaria. 4

A los fines de dar cuenta de lo planteado, apelaremos a la información contenida en periódicos y suplementos especializados circulantes a nivel regional, centrándonos en la columna institucional de la SRRC. El análisis de estas fuentes es relevante, puesto que son un medio habitual de 'mediatización de la ruralidad' al tiempo que se erigen como un espacio simbólico que supone la re-producción de las representaciones de los sectores agrarios (Carniglia, 2011). Estos soportes constituyen selecciones de datos de la 'realidad', que se organizan de determinada manera para decir, hacer entender y dar a conocer (Carreras Doallo, 2012) y, por lo tanto, son un elemento privilegiado para el análisis de las estrategias de los actores agrarios que contribuyeron a internalizar los imperativos del nuevo modelo productivo. A pesar del creciente interés que se registra en el ámbito de las ciencias sociales por desentrañar los diversos aspectos implicados en los proyectos editoriales, $\underline{5}$ el análisis de los soportes que abordan temáticas agrarias continúa escasamente explorado, aunque no se desconozca el 'valor' heurístico de estas publicaciones. De hecho, son empleadas habitualmente como fuentes primarias que permiten obtener datos significativos para reconstruir los entramados en los que se ven involucrados los actores agrarios. $\underline{6}$ A pesar de esto, existen valiosos antecedentes que para diferentes temporalidades han trabajado tanto respecto de las representaciones que sobre diversas cuestiones agrarias fueron plasmadas en revistas especializadas como en relación con el tratamiento periodístico que recibieron ciertas problemáticas, como los instrumentos impositivos que gravaron la tierra, la incorporación de tecnología en los procesos productivos, el lugar de la mujer en el campo y la familia rural, entre otras cuestiones (Gutiérrez, 2002, 2004 y 2005; De Arce, 2009 y 2011; Carniglia, 2011). En ese marco, fueron menos transitadas tanto las prácticas editoriales de las asociaciones de productores como los temas y problemas contenidos en sus páginas. Una excepción a esta situación lo constituyen los trabajos de Poggi (2011a y 2011b) donde se analizan las representaciones construidas por la SRA y FAA sobre la cuestión de la reforma agraria desde sus publicaciones. Estos trabajos nos habilitan a establecer puntos de contraste y comparaciones y, fundamentalmente, a observar la construcción histórica de los discursos que sobre el agro circularon en determinados momentos históricos.

\section{“Cada día más empresarios”: la construcción de un nuevo horizonte discursivo}

Desde fines de la década de 1980 la SRRC emprendió un conjunto de acciones que redefinieron su tradicional función gremial. Estas actividades consistieron en dotar a su estructura institucional de una amplia serie de servicios entre los que se destacaron la creación de rondas de negocios, la firma de convenios con empresas proveedoras de insumos y la estructuración de múltiples actividades de capacitación y actualización empresaria. Paralelamente, la dirigencia agraria introdujo una serie de cambios institucionales a partir de los cuales motorizó esas acciones, por ejemplo: se crearon delegaciones zonales en localidades aledañas, se constituyeron cámaras dentro de la entidad que tenían como función principal la de asesor en aspectos puntuales a la comisión directiva, se re-fundó el Ateneo Juvenil, etc.. ${ }^{7}$ Como contrapartida se pudo observar que las formas discursivas de interpelación que la dirigencia ruralista históricamente empleó se combinaron con otras tendientes a estimular el cambio en pautas y comportamiento empresario de sus bases sociales.

Teniendo en cuenta lo enunciado, en este apartado exploraremos dos de esas modalidades desde las que se construyó un nuevo horizonte discursivo en consonancia con aquellas coordenadas a que el nuevo paradigma productivo le otorgaba centralidad. Por un lado, examinaremos los sentidos que asumió la idea de reconversión productiva en la discursividad generada por la dirigencia de la SRRC. Aquí procuraremos dilucidar si se generaron tensiones en los principios y valores que tradicionalmente habían caracterizado a la 
entidad con la incorporación de tópicos asociados a una matriz de tipo tecnologizante (Balsa, 2007). Por otro lado, analizaremos las dimensiones que asumió la construcción de una estrategia de difusión del nuevo imaginario productivo. Frente a la intensidad que revistieron las acciones de oferta de servicios, capacitación y actualización de las bases sociales, la SRRC dispuso parte de su esfuerzo institucional en la publicación de una columna editorial en el suplemento agropecuario del diario local. A partir de esta estrategia de mediatización de las acciones institucionales se evidenciaron y cristalizaron operaciones discursivas tendientes a fijar la legitimidad de una nueva concepción de la actividad agropecuaria. Fue en ese proceso que quedaron expuestos -discursivamente- algunos de los desplazamientos y rupturas más característicos del agronegocio.

\section{"La reconversión, divino tesoro": $\underline{\underline{\beta}}$ una nueva forma de interpelación de las bases sociales de la SRRC}

Durante la década de 1990 emergía como enunciado operativo la ‘reconversión’ para explicar los procesos de transformación de los sujetos agrarios y de sus prácticas. Este era un término que atravesaba las interpretaciones de la totalidad del sector y era comúnmente utilizado tanto por los agentes de la Secretaría de Agricultura, Ganadería y Pesca (SAGyP) como por los dirigentes de las entidades rurales. Para los primeros, suponía parte del diagrama de una política agropecuaria destinada a aumentar la productividad y mejorar la competitividad del sector. $\underline{9}$ Para los segundos, el término remitía, además, a la necesidad de asumir una actitud más empresaria en la gestión de los procesos productivos. Ambos usos proponen pautas y modelos de comportamiento en cierta medida comunes pero, en el caso de los productores agropecuarios riocuartenses, su sentido se iría redefiniendo a partir de la dinámica económica y política de determinadas coyunturas. Por ejemplo, pudimos observar cómo la idea de reconversión era empleada en el momento de emergencia y de consolidación del nuevo régimen de acumulación para reclamar al Estado la habilitación de líneas de crédito que hicieran posible la reproducción de las unidades productivas en un contexto de declive de los precios internacionales. Asimismo, desde mediados de la década de 1990 la reconversión fue esgrimida como crítica a los partidos políticos y a los políticos para marcar la necesidad de que estos actores cambiaran sus prácticas tal como lo habían hecho los productores agropecuarios. $\frac{10}{10}$

Siguiendo esta cuestión, aquí nos interesa observar qué sentidos, trasladados al plano productivo, asumió la noción de reconversión en los discursos de la dirigencia de la SRRC. Hay en el discurso generado por los directivos de la entidad la construcción de la 'reconversión' como un imperativo, es decir, como una condición sine qua non que posibilita la salida del ‘estancamiento’ por el que atravesaba el sector a inicios de la década de 1990: "La necesidad de reconvertir es impostergable. La importancia deriva de la crisis de rentabilidad que vive el sector; la urgencia, dado los serios peligros de continuar como hasta ahora” (AHMRC, H, P, sábado 04/09/1993, p. 2-3). $\underline{11}$ Esa transformación no solo es inevitable sino que es, al mismo tiempo, una operación radical, puesto que conlleva asumir la obsolescencia de saberes que habían caracterizado los procesos productivos por décadas y constituían un capital social que se transmitía de generación en generación: el oficio de productor agropecuario (Hernández, 2009; Muzlera, 2009). Así quedaba implícito en el discurso de otro de los dirigentes:

"Lo que queda claro es la necesidad de cambiar las empresas agropecuarias, de mejorarlas, como está ocurriendo con todas las empresas argentinas. Ello obliga, primero, a un propio cambio de mentalidad. Supone admitir que lo que fue válido en el pasado puede dejar de serlo hoy. Esto cambia radicalmente el modo de pensar y actuar” (AHMRC, H, TA, viernes 21/01/1994, p. 2-3).

En ese contexto, la 'reconversión' no suponía alterar la estructura de la distribución de la tierra que había caracterizado a la región, es decir, trabajar con mayores cantidades de superficie en propiedad en desmedro 
de las unidades productivas más pequeñas; ni un suplantar una producción por otra sino intensificar, por ejemplo, el uso de insumos industriales (AHMRC, H, P, sábado 04/09/1993, p. 2-3). El desafío consistía, para la dirigencia de la entidad, en elevar la productividad y rentabilidad de la producción, lo que se podía lograr por medio de la conjunción de diversas alternativas. La primera, apelando a diversas estrategias asociativas que permitieran reducir costos, mejorar posicionamientos en la comercialización e incrementar los ingresos de las unidades productivas. Así, la editorial de la entidad recomendaba: en primer lugar, el adelanto de compras de insumos en block, como semillas (agrícolas y forrajeras), agroquímicos, herbicidas e insecticidas, productos básicos para la elaboración de raciones balanceadas en el predio. La segunda sugerencia, consistía en apelar a la contratación conjunta de técnicos de las distintas áreas (agrónomo, veterinario, economista, contador, administrador, etc.), de mano de obra especializada para solucionar algunos déficits transitorios o para trabajos que no justifican personal permanente, de trabajos de maquinaria especializada, como labranza cero, la henificación, las pulverizaciones agrícolas. Por último, una tercera alternativa, era asociarse y así facilitar el uso de la maquinaria existente, comprar maquinarias y equipos nuevos, utilizar equipos ya instalados como mangas y bretes, secadoras de granos, moledoras-mezcladoras, básculas, etc. También era una herramienta sugerida para realizar trabajos de tercerización de tareas agrícolas y forrajeras; para recibir capacitación en áreas que no son contempladas habitualmente como la de manejo financiero y de comercialización; y para aumentar el valor agregado de la producción, comercializando productos directamente al consumidor(AHMRC, H, $P$, sábado 04/09/1993, p. 2-3).

La segunda alternativa partía de considerar la estructura de costos fijos de las unidades productivas - previa crítica a la 'presión fiscal' ejercida por el Estado $\frac{12}{2}$ y ponía el acento en optimizar los recursos ya existentes en las unidades productivas y recurrir al asesoramiento técnico para hacer frente a los procesos productivos:

"La posibilidad de reducir los gastos tranqueras adentro están ligados a controlar los costos administrativos, lograr el ordenamiento financiero, perfeccionar el uso de la información, adoptar prácticas agrícolas probadas y aplicar asistencia técnica en todas las áreas que afectan al negocio. En definitiva, la reconversión productiva se produce esencialmente trabajando distinto sobre las cosas que tenemos y conocemos y solo una vez superados los problemas actuales de creación de nuevos mercados y facilidades de comercialización de la producción, intentar nuevas alternativas de producción”(AHMRC, H, P, sábado 04/09/1993, p. 2-3).

Sobre esta dimensión era, en definitiva, donde radicaba el 'cambio de mentalidad' que permitiría sobrellevar airosamente un período de transformaciones estructurales para el agro. En una efusiva interpelación un dirigente de la SRRC se refería a la necesidad de emplear 'decisiones estratégicas' en la 'gestión' de las ahora devenidas 'empresas agropecuarias', donde la planificación, el cálculo y análisis de costos y, fundamentalmente, el asesoramiento financiero eran condiciones indispensables para el 'negocio':
"No son algo misterioso ni esotérico. Se saben, se conocen, otros sectores ya las aplican. Y una vez que se cuenta con las herramientas, hay que dar paso a la acción. Animarse a vencer la inercia. Empezar a analizar proyectos nuevos, con prudencia y sin atropellarse. Considerando todos los factores que intervienen, además de los productivos (financiación, comercialización, impuestos). No podemos seguir quedándonos en diagnósticos y análisis mientras las empresas se funden. No hay mucho más para analizar. Hay que dejar la deliberación improcedente para pasar, con planes y prudencia, a la acción” (AHMRC, H, TA, viernes 21/01/1994, p. 2-3).

La construcción de estos enunciados que apelan a romper con hábitos y formas de producir que históricamente los productores agropecuarios habían asumido sin cuestionamiento y que se sostenían en un conocimiento de lo agropecuario basado en una experiencia ancestral implicó, en términos de Hernández (2009), una verdadera ruptura cognitiva. Ahora bien, este proceso no se produjo sin fisuras o tensiones ni 
constituyó un discurso unívoco en al interior de la SRRC. Para muchos productores agropecuarios el impulso dado a la reconversión había implicado asumir significativas inversiones, fundamentalmente, destinadas a la renovación de implementos y maquinaria agrícola. Muchos de estos gastos se habían afrontado asumiendo obligaciones crediticias (en instituciones financieras del sistema público y privado) que no pudieron ser canceladas. Esta problemática no parece haber afectado a la base social de la SRRC, puesto que no figura entre las principales reivindicaciones y demandas de la entidad como sí es central en el caso de la FAA. $\underline{13}$ Sin embargo, parte de la dirigencia de la SRRC se mostraba crítica frente a los 'esfuerzos’ que había significado la reconversión en términos de endeudamiento financiero producto de la carencia de una "clara política agropecuaria” (AHMRC, H, P, lunes 02/01/1995, p. 31).

Tal vez la crítica más vehemente provino del entonces presidente de CARTEZ y expresidente de la SRRC Eduardo Budd. En una editorial denominada "Reconversión, divino tesoro" el dirigente ruralista sancionaba la actitud de los funcionarios y ‘tecnócratas’ del gobierno nacional para quienes la reconversión constituía:
“(...) una nueva panacea y la solución mágica a todos los problemas que nos acosan (...) ¿Quién será entonces el iluminado que piensa que la reconversión puede poner fin a la crisis del campo? Sería interesante conocer qué es la reconversión en el sector agropecuario para estos funcionarios y tecnócratas que sin el más mínimo atisbo de sentido común llevan computadoras, con modelos y con números que en el papel dan bien y en el bolsillo mal (...) Que un ministro mande a los productores a 'vivir al campo para ahorrar' y un tecnócrata los saque 'tranqueras afuera' para hacer marketing no va, esto es desorden, desprolijidad y porque no decirlo ‘ignorancia'”(AHMRC, H, $P$, lunes 02/01/1995, p. 31).

Seguidamente esgrimía una crítica a la clase política que no ha podido 'renovarse' como si lo logró el 'campo', el cual “(...) se renueva constantemente y a lo largo de los años ha mejorado sus rendimientos en todos los subsectores. Hasta en algunos casos ha superado rindes internacionales con costes menores" (AHMRC, H, P, lunes 02/01/1995, p. 31). Como se desprende de este fragmento, el 'campo' en la perspectiva del dirigente ruralista era un sector que ya se había reconvertido y que necesitaba 'señales' por parte del gobierno nacional. Esas 'señales’ consistían en los clásicos reclamos históricamente sostenidos por la entidad: generar una política agropecuaria con una actitud agresiva en la "búsqueda permanente de mercados” y la reducción de las cargas impositivas que no retornaran en obras y servicios para el sector (AHMRC, H, P, lunes 02/01/1995, p. 31).

A pesar del impulso institucional que se le otorgó a los procesos de profesionalización de los perfiles de las bases sociales de la SRRC (Carini, 2014a), realizados bajo las coordenadas del agronegocio, el discurso relativo a los mismos distó de constituir un relato monocorde. Las diferentes coyunturas resignificaron el contenido de lo que para la dirigencia constituía la reconversión. De imperativo impostergable a panacea o solución mágica, las nuevas formas de concebir la producción agropecuaria como negocio generaron tensiones en el plano discursivo entre los principios liberal-conservadores en los que históricamente la dirigencia de la entidad había cimentado sus interpretaciones de la realidad y los enunciados asociados a la matriz tecnologizante a los que el contexto social y económico otorgaba centralidad. Es decir, lejos de constituir un plasma que bañó todas las interpretaciones de la época, profesionalización, empresarialidad, tecnificación fueron, en el plano discursivo, elementos que se incorporaron a veces conflictivamente a la discursividad de la entidad. Los enunciados, frases y expresiones asociados a la matriz discursiva liberalconservadora continuaron siendo el recurso expresivo más frecuentemente utilizado para interpelar y movilizar a las bases sociales. 


\section{La prensa como instancia de profesionalización de las bases sociales y legitimación del agronegocio}

Los vínculos entre prensa, agro y entidades gremiales fueron frecuentes y, por ende, poseen una dilatada historicidad. Muchas de las organizaciones rurales 'nacionales' estructuraron publicaciones destinadas a dar cuenta de las diferentes dimensiones vinculadas a su devenir institucional. Tal vez los ejemplos más conocidos sean los casos de Anales, publicación de la SRA, y el periódico La Tierra editado por la FAA. $\underline{14}$ La SRRC también mostró inquietud por mediatizar su quehacer institucional. Fue a partir de la década de 1990 que se observó un esfuerzo sostenido en este sentido. Más precisamente, desde el 29 de abril de 1994 y con una periodicidad semanal, se comenzó a publicar una columna institucional denominada Información Ruralista. Las motivaciones de esta iniciativa eran claras, así se lo reflejaba en la primera aparición: "Nuestra Sociedad Rural se ha comprometido brindar, a sus asociados y productores en general, una gama de servicios, que tienen que ver con su desenvolvimiento empresario y con su necesaria permanente actualización (AHMRC, H, SA, viernes 29/04/1994, p. 4). De esta manera, se emprende una actividad difusora no solo de las actividades institucionales de la SRRC sino de todo un conjunto de saberes y prácticas vinculados a una nueva concepción de agricultura. La lectura de Información Ruralista ofrecía a los productores, por ejemplo, información novedosa sobre las modificaciones en las obligaciones impositivas y previsionales, las nuevas líneas de créditos bancarios, las perspectivas para el comercio agropecuario, la política forestal, la vacunación y otras prácticas asociadas a la sanidad animal, recaudos para combatir plagas y malezas, los rendimientos de cosecha, la incorporación de tecnología de punta a los procesos productivos. La columna usualmente se puede ubicar en el género informativo. Sin embargo, ocupa un lugar relevante las notas del género de opinión, en especial, aquellas que se corresponden a editoriales y a análisis y/o comentarios.

Una de las particularidades que revistió esta empresa editorial fue que se incorporó como un espacio editorial dentro del Suplemento Agropecuario Tranquera Abierta del diario Puntal. $\underline{15}$ Es decir, a diferencia de los casos anteriormente citados, la columna de la SRRC se dirigió no solo a los asociados sino también a otros productores agropecuarios y al público interesado en temáticas rurales. Se ha señalado que los periódicos y/o suplementos rurales se dirigen también a los actores de la ciudad, un ámbito siempre clave de la decisión sobre aspectos básicos del trabajo, los consumos y la vida rurales (Carniglia, 2011, p. 161). A esto cabe agregarle que, es en ese ámbito, el de la ciudad, donde se dirimen las políticas públicas que se destinan al sector. Entonces, esta inclusión le posibilitó a la dirigencia ruralista ampliar el alcance de sus intervenciones y pudo desde ese lugar sentar un ‘deber ser’ sobre la actividad agropecuaria.

En este sentido, consideramos que la columna Información Ruralista funcionó como una plataforma que no solo permitió a la entidad abrir un canal de comunicación con sus bases sociales y, de esa forma, retener, fortalecer y estrechar su acción gremial, sino que, al mismo tiempo, favoreció una profesionalización al propiciar que adoptasen una 'actitud más empresaria'. Simultáneamente, la edición de la columna operó como un vehículo que permitió una determinada construcción simbólica sobre lo rural. Así, los discursos e interpretaciones esgrimidas por la dirigencia de la SRRC constituyeron una de las formas de penetración y legitimación de las prácticas y saberes ligados al nuevo paradigma productivo.

Bajo esta concepción, en este apartado atenderemos al entramado simbólico que la entidad construyó respecto del agronegocio. Exploraremos dos dimensiones discursivas que marcan una ruptura respecto al modelo de desarrollo rural previo. La primera se vincula con la dinámica generada por el nuevo paradigma socio-productivo en relación con las lógicas territoriales y la presencia de nuevos actores en el paisaje agrario. La segunda hace referencia al conjunto de saberes y habilidades a partir de los cuales la SRRC construyó un imaginario sobre el oficio del productor agropecuario y la actividad agropecuaria. 


\section{Las lógicas territoriales del agronegocio y los nuevos actores en la pampa riocuartense}

Como lo mencionamos previamente, uno de los elementos más visibles que trajo aparejado la configuración del agronegocio fue la acentuación del proceso de agriculturización, el cual, generalmente, estuvo asociado a la expansión de la soja. Este fenómeno revistió en el espacio económico de Río Cuarto un crecimiento geométrico que, si bien no alcanzó para desplazar completamente actividades productivas históricamente características de dicho espacio, sí ocasionó múltiples consecuencias socio-territoriales. $\underline{16}$ No obstante, la sojización (y sus implicancias) constituyeron un aspecto que preocupó a los empresarios nucleados en la SRRC. La inquietud de la dirigencia se asociaba a la conservación de las propiedades edafológicas de los suelos pero los señalamientos realizados no apuntaban a deslegitimar la vigencia del agronegocio sino que, por el contrario, se apelaba a una estrategia discursiva que apuntaba a una atenuación de los argumentos vertidos en su contra (Poggi, 2011). Es decir, se incorporaban en el discurso generado por la entidad para remarcar las características positivas que posee una determinada práctica o elemento a través de citas o referencias directas a estudios o a determinados agentes. En Información Ruralista se advertía sobre el peligro de la pérdida de las cualidades de los suelos producto de la intensidad que había asumido la agricultura y se esgrimía la necesidad de incorporar la siembra directa como paliativo de esa situación, cuestión que era apoyada por medio de un estudio del INTA. Desde la columna institucional se comentaba que:

"Hoy, esos suelos tienen menos de la mitad de la materia orgánica y de nitrógeno que cuando
se comenzó con la agriculturización permanente. El círculo vicioso de la degradación de los
suelos y el monocultivo no es fácil de interrumpir, porque juegan factores de tanto peso como
la estructura minifundista de la zona y la rentabilidad de los campos. Más hoy en día, cuando
muchos campos están siendo alquilados y explotados por terceros. Esta situación limita la
adopción de prácticas que frenen la degradación de los suelos - como pueden ser las prácticas
conservacionistas y la inclusión de cultivos con rastrojos voluminosos, como el maíz y mucho
mejor si esos campos fueran de producción mixta. Hemos mencionado el INTA, organismo
que ha hecho y sigue haciendo mucho por la conservación del suelo" (AHMRC, H, TA,
viernes $04 / 07 / 97$, p. 7)..$\underline{17}$

Asociado al proceso de agriculturización en la región en otras editoriales la entidad señalaba la preocupación por la situación de la ganadería, producción que históricamente había caracterizado a Río Cuarto y sobre la que se asentada todo el entramado socio-económico de la región (Carini, 2014a). Se denunciaban los procesos de desplazamiento que estaba sufriendo dicha actividad respecto de la agricultura por la coyuntura favorable de precios internacionales que atravesaba ésta última. El panorama que se trazaba era "francamente negativo”, evidenciándose una disminución de la producción de carne hacia zonas marginales dentro del departamento de Río Cuarto, una caída en los niveles de preñez y, en virtud de esto, en la producción de terneros desmejorando así la calidad de esa producción. Seguidamente la editorial empleaba enunciaciones, expresiones y frases correspondientes a dos matrices discursivas diferentes. Por un lado, se cuestionaba la “falta de previsibilidad de las autoridades” y, consecuentemente, de una política sectorial, reclamándose:

“(...) mejores condiciones para lograr esa mayor producción, como la implementación de líneas de créditos accesibles, con tasas razonables, que estén de acuerdo con la rentabilidad del sector. Por ahora, esto no solo no sucede así, sino que, por el contrario, los costos de esas empresas ganaderas aumentan día a día, desmejorando aún más su situación” (AHMRC, H, $T A$, viernes 04/10/96, p. 7).

Esta crítica formaba parte de la matriz discursiva de tipo liberal-conservadora que había caracterizado a la SRRC. Por otro lado, con esa discursividad comenzaban a percibirse enunciados que se pueden inscribir a 
una matriz tecnologizante. Los dirigentes de la entidad ponían de manifiesto que “(...) pese a todo los ganaderos perseveran y hacen esfuerzos para mejorar su producción, acudiendo a la implantación de pasturas adecuadas, con un manejo racional y sin descuidar su infraestructura e instalaciones" y recordaba que "La Cámara de Ganadería de esta Sociedad Rural brinda su servicio de asesoramiento y actualización técnica como contribución a ese mejoramiento productivo ¡Acérquese a nuestra sede!” (AHMRC, H, TA, viernes 04/10/96, p. 7).

Como ya lo dijimos, el llamado para que los productores se acercaran a la SRRC se vinculaba con la estructuración de estrategias de empresarialización de las bases sociales que la entidad llevaba a cabo desde fines de la década de 1980. Claramente se exponían estos lineamientos en una nueva columna institucional. Se pueden identificar en el desarrollo editorial - como en el caso anterior - la complementación de elementos de la matriz liberal-conservadora y de la tecnologizante. Por una parte, se da cuenta de la centralidad del sector agropecuario para el crecimiento de la economía nacional y regional:

\begin{abstract}
"Las expectativas de siembra de granos gruesos: maíz, sorgo, girasol, soja, maní, etc. de acuerdo con estimaciones oficiales y privadas, son realmente alentadoras. Nuestro sector está nuevamente dispuesto a brindar su importante cuota, en favor de la economía regional y nacional, contribuyendo decididamente a la reactivación de nuestras comunidades. Los comercios del ramo venderán más semillas, más insumos, más herramientas de trabajo, más maquinaria agrícola, más de todo, poniendo de manifiesto la importante gravitación del sector agropecuario en el desenvolvimiento integral de pueblos y ciudades” (AHMRC, H, TA, viernes 27/09/96, p. 7).
\end{abstract}

Y, por otra parte, se argumentaba sobre la necesidad de asumir una mentalidad empresarial que implicaba recurrir al asesoramiento profesional en tanto aspectos productivos como administrativos y, fundamentalmente, a la incorporación de los avances en el ámbito de las tecnologías y los implementos agrícolas.
“Quizá la circunstancia sea nuevamente propicia, también, para reflotar algunas sugerencias: acudir, en la medida de las posibilidades, a la moderna tecnología, realizando una buena inversión, a favor de precios compensatorios de los cereales y oleaginosas. Utilizar buena semilla, realizar una adecuada implantación del cultivo elegido, seguir su evolución como corresponde, emplear maquinaria e implementos adecuados e ir pensando en la comercialización de esa producción que esperamos. ¡Hacerse cada día mejores empresarios! Acudir a los profesionales técnicos, a la asesoría contable y fundamentalmente A LAS ENTIDADES QUE LOS REPRESENTAN! Esta Sociedad Rural, a través de sus asesores, de sus cámaras adheridas, de convenios de integración, con organismos y otras entidades, está en la tarea de apoyar esa necesaria actualización de los productores ¡TENGAMOS EN CUENTA... ESTAMOS A SU DISPOSICIÓN!” (AHMRC, H, TA, viernes 27/09/96, p. 7. Mayúsculas en el original).

Otro componente clave implicado en las lógicas del agronegocio fue la incorporación de tecnología y la generación de nuevas solidaridades como las que se expresaron por medio de los pools de siembra y de la vinculación con agentes financieros extra-locales. En este punto, resulta interesante rescatar la argumentación sobre los 'beneficios' de pools de siembra en la región. La dirigencia realizaba una extensa comunicación en la que procuraba "desmitificar" los "presuntos aspectos negativos" de estos actores y remarcar "algunos resultados muy auspiciosos” de los mismos. En primer lugar se enfatizaba en el componente local que muchas de esas empresas poseían y también en el movimiento económico que generaban para la ciudad, puesto que se abastecían de insumos en comercios locales, muchos industrializaban los cultivos en plantas procesadoras de la región y “(...) el cincuenta por ciento de lo 
producido queda en la región. Se suma al alquiler de los campos, el monto de los costos por las labores que se realizan mediante los contratos respectivos" (AHMRC, H, TA, viernes 26/04/96, p. 7). En segundo lugar, se abogaba por la función que cumplían estas empresas en cuanto a evitar la salida de los productores del proceso productivo aunque no se lograba dimensionar que esa situación en muchos caos - como ha sido señalado por la literatura especializada -era irreversible. La columna recordaba que:

“(...) son muchos los productores de la zona, que contratan el alquiler de parte de sus campos con estas empresas, comprometiendo su propio trabajo pero, sin endeudarse, con un saldo económico importante y favorable. A través de estas asociaciones, favorecen la permanencia de los productores en sus predios, evitando que sean expulsados del sistema, lo que es bueno para las economías regionales. De lo contrario, muchos de esos productores pasarían a engrosar poblaciones urbanas, en éxodo proclive al pauperismo y a los problemas sociales propios de las grandes urbes" (AHMRC, H, TA, viernes 26/04/96, p. 7).

Por último, en tercer lugar, la columna destacaba una suerte de paradoja a los fines de legitimar la presencia de los pools de siembra en la región. Lejos de entender que estas actividades eran intensivas y que podían en ocasiones - comprometer la sustentabilidad de los suelos y los recursos naturales se sostenía que implementar las modernas tecnologías de labranza y recurrir a un adecuado asesoramiento técnico producían el efecto contrario:

\begin{abstract}
"En lo que hace a la conservación de los suelos, hay que tener en cuenta que muchas de estas empresas trabajan con métodos conservacionistas, con técnicas adecuadas para la preservación de los recursos naturales - por ejemplo, la siembra directa - incorporando a los establecimientos rurales afectados por estos contratos, maquinaria agrícola y tecnologías de última generación. Igualmente es necesario advertir que estas empresas cuentan con el asesoramiento de profesionales técnicos de conocida capacidad e idoneidad, que siempre dejan enseñanzas en cuanto a los más eficientes sistemas de trabajo y de producción. Por último, cabe reflexionar, con relación a los condicionamientos que nos impone esta dura economía, no solo de Argentina, sino de todo el mundo moderno, que muchos productores por si solos no pueden hacer frente a todas estas exigencias, de viendo apelas a estos convenios con los pools de producción, para sortear estas dificultades. En suma: hay que aceptar las realidades y obrar en consecuencia”(AHMRC, H, TA, viernes 26/04/96, p. 7). $\underline{18}$
\end{abstract}

En síntesis, a partir de las intervenciones en la columna institucional, la dirigencia de la SRRC construyó una legitimación de las lógicas implicadas en el agronegocio. A pesar de que se avizoraban algunas problemáticas derivadas de este nuevo ordenamiento productivo, las críticas esgrimidas no llegaban a cuestionar la vigencia del mismo. Como contrapartida, se tendió a enfatizar en los beneficios de las nuevas tecnologías y de la actividad económica de los nuevos actores.

\title{
La empresa innovadora y el nuevo empresario rural
}

Se ha sostenido que la vigencia del agronegocio implicó una serie de desplazamientos en relación con las formas de concebir la actividad agropecuaria que se tradujeron en modalidades radicalmente distintas respecto de etapas anteriores de operar dentro del proceso productivo (Hernández, 2009; Gras y Hernández, 2009 y 2013a). Mirados en conjunto, estos procesos acentuaron el carácter empresario de la actividad agropecuaria, la complementación de habilidades y saberes de diferentes ámbitos del conocimiento y la necesaria integración de lo rural con lo industrial. En ese marco, articulado en la idea de la reconversión empresaria, desde Información Ruralista se comenzaban a realizar referencias a esos desplazamientos y, por ende, a socializar un conjunto de saberes y prácticas vinculados al agronegocio. Un lugar destacado lo 
ocupaba la necesidad de procurar un manejo empresarial de las explotaciones agropecuarias como plataforma necesaria no solo para alternar exitosamente en los mercados internacionales sino también para asegurar su reproducción. $\underline{19}$ Así, las instancias de profesionalización de la actividad agropecuaria generadas por la SRRC poseían su contrapartida en la faz discursiva que alertaba sobre las mutaciones en el negocio agropecuario y, por ende, en la institucional:

“(...) por cuanto Argentina está inmersa en un mundo que ha cambiado. Esta Sociedad Rural de Río Cuarto continuará ejerciendo su democrática posición de defensa del sector agropecuario (...) Pero también sabemos que nuestra acción deberá encaminarse al mejoramiento de [la] vida de nuestras empresas rurales”. (AHMRC, H, TA, viernes 24/07/1998, p. 7).

Esta suerte de toma de conciencia de la dirigencia rural se asentaba discursivamente en el imperativo de garantizar una transformación en las estructuras productivas de las empresas agropecuarias de los productores asociados a la SRRC que ahora debía orientarse a incrementar la productividad a través de la incorporación de tecnología y a realizar un uso "inteligente” de las habilidades administrativas y financieras.

\begin{abstract}
"Ya nadie puede estar ajeno a la profunda transformación que viene dándose en los sistemas agroalimentarios. Este deviene, indudablemente, como consecuencia de la creciente globalización de las economías mundiales, modificando las estructuras productivas, industriales y fundamentalmente comerciales de todos los países, que ya no admiten posicionamientos que pretendan asimilarse o no involucrarse en estos cambios. (...) Las empresas rurales se ven obligadas a producir cada días más, para lo que deben adecuar sus estructuras, actualizándolas y preparándolas para esa mayor producción. Ello significa también la necesidad de acceder a la moderna tecnología e implica necesariamente una mayor capitalización. Pero ésta debe ser racional, ordenada y oportuna, de acuerdo con la propia envergadura de cada empresa. No siempre esa tecnología implica grandes inversiones, pero sí alcanzar una estructura adecuada, que permita los cambios que deben darse en el andamiaje de la empresa, preparándola para acometer el desafío de nuestros tiempos” (AHMRC, H, TA, viernes 24/07/98, p. 7).
\end{abstract}

Finalizaba la editorial afirmando que se estaba en presencia de una "verdadera revolución [que] se da no solamente en los sistemas productivos. Esto se continúa, tiene una natural correlación, en el desenvolvimiento empresario" (AHMRC, H, TA, viernes 24/07/98, p. 7). Esta noción implicaba, para la dirigencia rural de la SRRC, hacer un uso adecuado de las opciones que ofrecen los mercados y recurrir a “una buena y oportuna asesoría” (AHMRC, H, TA, viernes 24/07/98, p. 7). Sin embargo, lo anterior no nos debe llevar a pensar que los elementos del discurso tecnologizante fueron apropiados de forma plena por la dirigencia rural de la entidad. Por un lado, como detallamos, se insistía en prestar atención a los "nuevos requisitos" de la actividad agropecuaria, mejorar eficiencia y productividad a partir de los avances tecnológicos. Por el otro lado, existían en la columna institucional referencias constantes a ser cautelosos con endeudarse más allá de los límites que permitía la propia empresa. Esta situación se ponía de manifiesto especialmente con la cuestión de la tecnología, que se consideraba como un "espejismo”:

“¡Cuánta cosa buena que hay para mejorar nuestra producción agropecuaria! Empero, debemos ser cautelosos a la hora de tomar decisiones, Los productores ya lo saben, pero siempre es oportuno formular alguna prevención o reflexión: mucho de lo que apreciamos en las parcelas agrícolas demostrativas, no deja de ser un espejismo. Son cultivos o pasturas logradas así con recursos que no se tienen en el campo: riego, encanalado del suelo, fertilizadas al máximo, con cuidados a lo largo de su evolución, que bien merecen el calificativo de 'bebes malcriados'. Con 'todos los chiches', por adoptar una expresión popular. Esa tecnología, adaptada a nuestras reales posibilidades, podrá ser asimilada paulatinamente, 
como sucede con todos los adelantos, pero no debemos pretender tener lujos inmediatamente. ¡No nos dejemos encandilar! En cuanto a la maquinaria agrícola, también hay que contemplar su eventual adecuación al dimensionamiento de nuestro campos y a nuestras necesidades iy posibilidades!” (AHMRC, H, TA, viernes 21/03/97, p. 7).

Cobraba relevancia en ese contexto, la apelación a estrechar vínculos transectoriales que atendieran a una diversificación de la red de producción en virtud de las cualidades-especificidades de consumidores ubicados en los más diversos mercados internacionales. Recordemos que uno de los pilares del nuevo modelo agropecuario lo constituyó la posibilidad de trasvasar las fronteras de lo rural e integrar distintos actores y componentes de la cadena productiva que agregaran una mayor complejidad al negocio agrícola (Hernández, 2009; Gras y Hernández, 2013). Para la dirigencia de la SRRC, esto se traducía un llamado para que sus asociados incrementaran su empresarialidad, procurando el desarrollo de emprendimientos agroindustriales.

\begin{abstract}
“(...) debemos pensar en emprendimientos que permitan procesar la materia prima que se obtiene en nuestros campos. Favorecer la radicación de agro-industrias sobre la base de los productos alimenticios que tenemos a nuestro alcance. Río Cuarto, insistimos, es un importante - y natural - centro dinamizador de la economía regional, con base agropecuaria. Es un núcleo comercial proveedor de insumos para el campo de una dilatada región. Pero, ha llegado la hora de pensar en la transformación de nuestra producción primaria, en el procesamiento de esos bienes en la industrialización. Más precisamente en la agro-industria. Procesar, en el lugar en el que se da la producción, incorporándole mano de obra e inversiones de capital, procurando un mayor movimiento económico general. He aquí, entonces, el gran desafío de la hora actual” (AHMRC, H, TA, viernes 08/03/96, p. 7).
\end{abstract}

Otro núcleo de la discursividad generada desde la columna institucional de la SRRC fue el que se abocó a comentar las competencias y saberes que se debían considerar para enfrentar la actividad productiva como un negocio. En este sentido, se generó un discurso con una alta performatividad empresaria, es decir una enunciación que legitimaba prácticas que consideraba adecuadas en el nuevo contexto productivo y que pretendía generar un cambio en las modalidades de operar en el proceso productivo de las bases sociales de la entidad. En ese discurso la eficiencia constituía la coordenada sobre la cual se asentaba la garantía de la reproducción de la empresa agropecuaria. Esto implicaba no solo atender a mejorar las técnicas y demás aspectos atinentes a lo productivo sino también perfeccionar lo relativo a la comercialización de la producción. En ese proceso, resultaba esencial el asesoramiento especializado, tal como lo podemos apreciar en el siguiente fragmento:

"Si nos sentimos eficientes, debemos procurar serlo más, mejorando nuestros sistemas de
producción, delineando estrategias superadoras de la actual situación, con vistas a mejorar
rendimientos que, a su vez, nos permitan acceder a una mejor posición vendedora de lo que
producimos. Debemos estar alertas e informados sobre el desenvolvimiento, las perspectivas y
la evolución de los mercados. Deberemos practicar mejores técnicas de producción. Ver lo que
hace el vecino y cómo lo hace, si es que éste obtiene mejores resultados que nosotros.
Escuchar a los técnicos que demuestran reales conocimientos y una sabia experiencia, con
resultados a la vista. Tener un panorama global. Estamos inmersos en un mundo cada vez más
competitivo y cada vez más exigente. No podemos aislarnos. Por el contrario, debemos actuar
en consecuencia.” (AHMRC, H, TA, viernes 18/11/1994, p. 7).

Así, desde el discurso de la dirigencia ruralista se comenzaba construir la necesidad de incorporar al conocimiento como un nuevo factor (decisivo) para llevar adelante el proceso productivo. Esta situación era advertida casi de forma constante en las páginas de Información Ruralista que al respecto comentaba:

“(...) los productores dedican sus esfuerzos para la adecuada y oportuna implantación de 
cultivos de cosecha gruesa (...) Estas tareas suponen inversiones, fuertes erogaciones sobre las que necesariamente deben tomarse decisiones empresarias racionales, suficientemente analizadas y evaluadas, dado que sobre este basamento económico-financiero, deben sustentarse todos los planes de producción del campo. Hoy en día, deben tenerse en cuenta todas las estimaciones que cada ejercicio requiere. En el caso de la agricultura, se deben tomar las lógicas y razonadas evaluaciones, sobre la base de datos actualizados de mercado y sus perspectivas, para lo que será indispensable una correcta información y una confiable asesoría. Así, al momento de adquirir semillas y agroquímicos, sin desdeñar el grado de confianza que puedan ofrecernos determinados operadores del mercado, deberemos analizar y evaluar precios, condiciones, calidad de la mercadería, financiamiento, etc. ya que el comercio dispone ahora de numerosas líneas, al alcance de cada productor. Se deberá disponer de una información actualizada sobre líneas de créditos, con sus respectivos plazos, intereses, montos prestables, etc. Será también necesaria la actualización técnica sobre rendimientos que ofrecen las distintas variedades, tareas culturales, ciclos de cada semilla, su aptitud productiva, su tolerancia frente a plagas, enfermedades, malezas, etc. En cuando a los agroquímicos, herbicidas, plaguicidas, fertilizantes, etc. Su debido conocimiento técnico sobre su correcta aplicación, etc. (...) creemos que la actualización, la información, el análisis y la evaluación económicos, son presupuestos que nadie debe desdeñar" (AHMRC, H, TA, viernes 10/10/97, p. 7).

De forma más enfática se sostenía: "Debemos hacernos cada día más empresarios. Acudir a la información actualizada, asesorarnos convenientemente, conocer la situación de los mercados” (AHMRC, H, TA, viernes 10/05/96, p. 7). Se argumentaba de esta forma sobre la necesidad de reconvertir la actividad agropecuaria en una de tipo empresarial y asumir roles más activos en relación con el conocimiento. El carácter empresario era asociado a la toma de decisiones basadas en manejo de información sobre tópicos que excedían lo meramente productivo. De esta manera se consideraba fundamental para el perfil socio-productivo del productor agropecuario la atención de los diversos componentes del campo financiero como la inevitabilidad de recurrir al asesoramiento de técnicos y especialistas en diversas áreas del conocimiento.

La actividad agropecuaria, para la dirigencia de la SRRC ya no consistía en el dominio de habilidades meramente agronómicas sino que incluía una vasta gama de competencias. Se trataba de una actividad cada vez más profesionalizada donde el conocimiento sobre la nueva coyuntura productiva y financiera requería de una actualización permanente con el fin de asegurar la performance empresaria de los productores. En un extenso fragmento que transcribimos a continuación, la dirigencia de la SRRC daba cuenta de esta ruptura y abogaba por la necesidad de abandonar el “conservadorismo” y asumir un "manejo empresario":

“Quizá por tradición, por conservadorismo o por su propia naturaleza, el productor ha sabido
manejarse siempre con capacidad e inteligencia en los diversos sistemas productivos, pero -
ahora más que nunca - muestras seria falencias en lo que hace a su manejo empresario ¿Cómo
se advierte esto? En un posicionamiento no del todo firme frente a los mercados, en donde
debe comercializar su producción. (...) Los mercados ya no son los mismos de algunos años
atrás. Se han diversificado. Actúan de manera distinta. En nuestros días se manejan múltiples
opciones para la venta de productos primarios o procesados, industrializados. En materia de
granaria, debemos conocer opciones, mercados de futuro, etc. en cuando a la ganadería, los
otrora grandes mercados concentradores van perdiendo su hegemonía y en cambio existe la
posibilidad de vender en campo, en ferias locales, etc. Debemos conocer todas estas variables
y operar con las que nos ofrezcan mejores posibilidades. Pero no solo debemos conocer todo
sobre comercialización. Debemos igualmente saber manejar los créditos, es decir los diversos
elementos de financiación de la empresa rural. En definitiva: escuchar a los que saben.
Asesorarse” (AHMRC, H, TA, viernes 19/04/96, p. 7).

Existía en la dirigencia una percepción sobre la necesidad de amoldar las prácticas de las bases sociales de la entidad a la nueva realidad productiva. En su discurso se encontraba presente la convicción de un quiebre 
respecto a la forma de concebir la actividad agropecuaria. Así, a diferencia de un pasado no tan lejano el éxito de la empresa rural ya no dependía de la habilidad de su titular para "manejarse en los distintos sistemas productivos”. Este tipo de conocimiento heredado, de generación en generación, como lo plantea Hernández (2009), se tornó superfluo. El imperativo del nuevo contexto productivo requería de la decisiva intervención de los saberes de técnicos y especialistas, la dirigencia de la SRRC entendía que ya no bastaba con el saber agronómico sino que apelaba a que éste fuera complementado con diferentes capacidades.

\section{A modo de cierre}

La profesionalización de la actividad agropecuaria iniciada por la SRRC demandó la necesidad de validar la adopción de nuevos saberes y habilidades. Esa operación fue realizada mediante la estructuración de una estrategia discursiva que tuvo una alta performatividad empresaria, puesto que procuró estimular el cambio en las pautas y comportamientos productivos de las bases sociales de la entidad, apelando a que asumieran las labores productivas como unas de tipo empresarial. Es decir, se propiciaba un quiebre en la concepción de la actividad agropecuaria. La construcción de este nuevo imaginario se mediatizó, fundamentalmente, a través de la prensa gráfica. Este soporte no solo posibilitó comunicar las actividades institucionales a las bases sociales sino que además le permitía a la dirigencia ruralista alcanzar a un público más amplio. Información Ruralista, la columna institucional de la SRRC se mostró como un mecanismo difusor eficaz para cimentar la profesionalización del oficio de productor agropecuario.

En esta operación se encontraba implícita una modalidad distinta de interpelación de las bases sociales que incorporaba componentes discursivos diferentes a los que históricamente habían caracterizado la enunciación de la entidad. La dirigencia de la SRRC acudió a enunciados, frases y conceptos de corte tecnologizante. Así, eficiencia, tecnología, cálculo racional, manejo empresario, asesoramiento, fueron las coordenadas desde las cuales se procuró producir un cambio en las formas tradicionales de concebir (y operar) dentro y fuera del proceso productivo. En un plano discursivo, para la dirigencia de la SRRC se trataba, fundamentalmente, de un cambio en las estructuras comerciales y productivas que volvían superflua la experticia meramente agronómica. Bajo esa idea rectora, por un lado se hacía hincapié en que los productores prestasen atención a las perspectivas y modalidades de negocios que proponían los mercados nacionales e internacionales. Esto era una pre-condición de la actividad productiva -tan importante como la fertilización de los suelos- que aseguraba la comercialización y, por ende, la rentabilidad de lo producido. Por otro lado, era preciso tener presente que la actividad agropecuaria constituía una cuestión de especialistas. Hubo una apelación constante destinada a remarcar el carácter excluyente que poseía el asesoramiento técnico. Éste no se limitaba solo a lo agronómico sino que abarcaba otras competencias como lo legal, lo contable, lo administrativo que necesariamente debían resolverse 'tranqueras afuera'. También en esa forma de concebir la actividad agropecuaria residía la convicción de que se debía tener en cuenta no solo lo que ocurría tranqueras adentro sino que cada vez ocupaba más importancia lo que acontecía por afuera, con la comercialización de la producción. Estas argumentaciones -de carácter constante- vertidas en la columna institucional constituían la contracara de un conjunto de conferencias, seminarios, demostraciones, capacitaciones, etc. que habían resignificado las funciones asumidas por la SRRC. De esta manera, el discurso operaba como un medio que reforzaba la idea del cambio que se estaba llevando a cabo en el modelo organizativo de la entidad y que también era indispensable reproducir en las explotaciones agropecuarias de los asociados a la entidad.

Estos elementos del discurso de la entidad respondían a una matriz discursiva tecnologizante, es decir, distinta a la que históricamente había orientado las intervenciones públicas de los dirigentes de la SRRC. Lejos de poner en tensión los principios sobre los cuales se habían asentado las interpretaciones de la dirigencia se incorporaron sin alterarlos y, en ese proceso, adquirieron un nuevo sentido. En ese entramado discursivo, los imperativos del nuevo modelo agrario aparecían como ineludibles. La reconversión 
empresaria era la noción (multifuncional) que se empleaba para marcar la impostergable necesidad de producir un cambio tanto en las prácticas como en las modalidades de concebir el proceso productivo.

Sin embargo, en la justificación del agronegocio a partir de los tópicos tecnologizantes realizada por la dirigencia de la SRRC pueden reconocerse ciertos límites. Se observaban las consecuencias negativas de la sojización pero se relativizaban sus efectos a partir de la adopción de la siembra directa, se denunciaba el desplazamiento de la ganadería por la agricultura pero se encontraban las causas en la inexistencia de una política agropecuaria, se celebraba el avance tecnológico pero se llamaba a cautela al momento de implementarlos al proceso productivo. Es decir, la dirigencia riocuartense -a similitud de lo que había acontecido con la performatividad política que proyectaba la convertibilidad (Carini, 2014)- realizaba señalamientos que ponían de manifiesto efectos adversos de las lógicas involucradas en el agronegocio pero que no llegaban a cuestionar la legitimidad que había adquirido entre los productores.

En definitiva, el posicionamiento de la SRRC frente a un contexto que implicaba profundas transformaciones en la dinámica económica y política supuso la necesidad de ofrecer una respuesta activa por parte de la dirigencia agraria para garantizar la propia reproducción institucional. Así, los cambios en los discursos y las prácticas fueron verso y anverso de una misma estrategia institucional por medio de la cual no solo se vehiculizó una determinada construcción simbólica sobre lo rural sino que, concomitantemente, se erigió además en un canal por el que penetraron y se legitimaron los saberes ligados al nuevo paradigma productivo.

\section{Notas}

1 Lattuada (2006), retomando una categorización de Moyano Estrada (1991) para el caso español, ha propuesto la presencia de dos tipos ideales de organizaciones en el agro pampeano: reivindicativas y no reivindicativas. A pesar de que ambas actúan como actores colectivos en los procesos de intermediación social se diferencian según la amplitud de objetivos que persiguen, los valores que poseen sus dirigentes y cómo los trasladan a sus discursos así como por la función que asumen respecto de su base social. Las primeras se caracterizan por tener como objetivo fundamental la defensa integral de (todos) los intereses de un determinado colectivo, ser universalista en la naturaleza de los fines que persiguen y en la actividad que desarrollan y tener un discurso con un fuerte componente ideológico, construido en función de los valores que asumen sus dirigentes y de las formas en que éstos interpretan los problemas de su base social. En tanto que las asociaciones agrarias no reivindicativas se caracterizan por la defensa de intereses no integrales de sus asociados (en general, aunque no exclusivamente, intereses de naturaleza económica), lo que implica que orientan sus acciones hacia una lista cerrada de temas o cuestiones, sus acciones presentan una naturaleza no universalista (exclusivista) y su discurso no es necesariamente ideológico, en el sentido de que no intenta ser una determinada visión del mundo ni una forma de interpretar los problemas generales de su base social (real y potencial), sino que es un discurso pragmático acotado no a valores, sino a un fin específico.

$\underline{2}$ Un estado de la cuestión exhaustivo sobre la emergencia de la nueva institucionalidad agraria y su implicancia sobre las dinámicas institucionales de las asociaciones tradicionales puede encontrarse en Carini (2017).

$\underline{3}$ Entidad de primer grado nacida en 1938, como efecto del accionar de la Agrupación de Accionistas Ley 11.747. Región Sud de Córdoba, la que entendía que había agotado los fines para los cuales había sido constituida y, por ende, era necesario profundizar la agremiación en otra entidad. Se encontraba adherida a Confederación de Asociaciones Rurales de la Tercera Zona (CARTEZ) y, por su intermedio, a la CRA. La 
SRRC nuclea a medianos y grandes productores, especialmente dedicados a la producción agrícola mixta, con un marcado predominio de la ganadera así como actividades vinculadas a la genética animal en cabañas. En cuanto al perfil social de sus asociados, la institución agrupaba mayoritariamente a profesionales (especialmente abogados, ingenieros agrónomos y médicos veterinarios), que poseían cierta diversificación de sus actividades (en muchos casos asociadas con la venta de servicios y productos agrícolas) y estaban ligados al poder político, especialmente al provincial y nacional. En cuanto los discursos que históricamente sostuvo se pueden inscribir en una matriz de tipo liberal-conservadora asociada a la defensa de la propiedad de la tierra, la no intervención del Estado en los negocios privados (Carini, 2014; 2014a y 2015).

4Sobre esa base, consideramos a la columna institucional como instrumento de legitimación que posee la facultad de producir y fijar legitimidades, es decir, de justificar prácticas y maneras de ver (Angenot, 2010). Al mismo tiempo, estas prácticas discursivas poseen una alta performatividad, es decir, capacidad para inducir cambios en las prácticas de los sujetos. Las construcciones simbólicas sobre la realidad agraria en su columna institucional son caracterizadas y analizadas a partir de la inscripción en lo que Balsa (2007, 2012 y 2013) ha identificado como las tres formaciones discursivas dominantes en los discursos públicos de las asociaciones agrarias. Al retomar el concepto de formación discursiva de Foucault (1968) la construcción de la tipología se asienta sobre la reiteración de enunciados, el empleo de ciertos términos y expresiones clave que le otorgan y construyen un sentido determinado sobre la cuestión agraria. En este sentido, se destacan las modalidades de interpelación y la construcción de los destinatarios del discurso, las representaciones sobre el Estado y su rol en la economía, la cuestión tributaria y la propiedad de la tierra. Si bien se encuentra implícito en el planteo de Balsa (2007, 2012 y 2013) y en el concepto propuesto por Foucault (1968), cabe destacar que estas formaciones discursivas poseen historicidad y pueden adquirir preponderancia unas frente a otras en determinadas coyunturas históricas. Esta cuestión reviste un interés central para nuestra investigación puesto que nos permite identificar cambios y continuidades en las formas históricas tanto de interpelar a las bases sociales de la entidad como de interpretar los problemas sectoriales.

$\underline{5}$ En la actualidad se evidencia un creciente interés por indagar los diversos aspectos implicados en las empresas editoriales de diferentes temporalidades donde resultan capitales los aportes de la antropología, la literatura y la historia de las ideas. Ocupó un lugar privilegiado en ese campo el estudio de los proyectos editoriales impulsados por revistas culturales, el análisis de la recepción y circulación de ideas y los públicos consumidores. También se han emprendido trabajos que abordaron las empresas editoriales o bien se enfocaron en estudiar los diarios de mayor circulación nacional.Realizar un estado de la cuestión sobre esta temática excede los límites de este artículo. Solo queremos mencionar algunos de los trabajos que contribuyeron a abrir estos interrogantes para la historia argentina del siglo XX, abordado textualidades específicas o que han planteado minuciosas síntesis y/o balances: Sidicaro (1993), Saítta (1998), Eujanian (1999), Girbal-Blacha y Quatrochi (1999), Ulanovsky (2005), Da Orden y Melón Pirro (2007), Lobato (2009), Lida (2012), entre otros.

6 Algunas reflexiones sobre estos tópicos pueden consultarse en Carreras Doallo (2012).

$\underline{7}$ Por razones de espacio no podemos ahondar en esta dimensión de análisis. Una primera aproximación la ofrecimos en Carini (2014a).

8 Archivo Histórico Municipal de Río Cuarto (AHMRC), Hemeroteca (H), Puntal (P), lunes 02/01/1995, p. 31.

9 Ver por ejemplo las declaraciones de Felipe Solá, responsable de la cartera durante el período 1989-1998. Discursos más extremos fueron esgrimidos, por ejemplo, por el ing. Jorge Antonio Ingaramo para quien "En la Argentina deben desaparecer 200.000 productores agropecuarios por ineficientes”. Bajo el enunciado de la reconversión, por ejemplo, se estructuraron desde el gobierno nacional un conjunto de programas para 
apoyar a las pequeñas y medianas explotaciones agropecuarias en el proceso de adecuación a la nueva realidad económica.

10 Remitimos a Carini (2014 y 2014a) donde abordamos con mayor profundidad esta cuestión.

11 Ver también: AHMRC, H, P, domingo 16/01/1994, p. 28.

12 Este era un argumento habitualmente presente en los discursos enunciados por la dirigencia en el acto inaugural de la feria de invierno y un componente central de la discursividad de tipo liberal-conservadora. Ver por ejemplo: AHMRC, H, P, domingo 10/09/1995, p. 24; AHMRC, H, P, domingo 01/09/1996, p. 25; AHMRC, H, P, domingo 14/09/1997, p. 24.

$\underline{13}$ Ver por ejemplo lo enunciado en Carini y Olivera (2016).

14 No existen abordajes exhaustivos sobre estos proyectos editoriales. Una excepción lo constituyen los trabajos de Poggi (2011a y 2011b) que desde la teoría crítica del discurso ha explorado algunas de las dimensiones en torno a las representaciones de la reforma agraria en las publicaciones de la SRA y FAA durante la década de 1970.

15 La edición del suplemento estuvo a cargo de quien al mismo tiempo se desempeñaba como jefe de prensa de la SRRC. Esta publicación circulaba los días viernes y presentó características bastante uniformes para el período que va entre mayo de 1995 y enero de 2002: su estructura o conjunto de secciones no varió y la cantidad de páginas osciló entre 8 y 12 de tamaño tabloide. Los promedios anuales por edición de la circulación neta pagada de los días viernes para el período considerado fueron de 6.197 ejemplares. Discriminado año por año del período 1994-2002 la edición neta pagada para los días viernes fue de: 1994: 5.039 ejemplares; 1995: 8.172 ejemplares; 1996: 7.211 ejemplares; 1997: 7.044 ejemplares; 1998: 6.635 ejemplares; 1999: 6.098 ejemplares; 2000: 5.062 ejemplares; 2001: 5.520 ejemplares y 2002: 4.995 ejemplares. Datos proporcionados por el Instituto Verificador de Circulaciones (IVC).

16 Remitimos a la lectura de Carini (2014a).

17 Ver también: AHMRC, H, TA, viernes 13/06/97, p. 7.

18 Ver por ejemplo: AHMRC, H, TA, viernes 26/04/96, p. 7; AHMRC, H, TA, viernes 10/05/96, p. 7.

19 Ver por ejemplo: AHMRC, H, TA, viernes 18/11/1994, p. 7.

\section{Referencias bibliográficas}

Alapin, H. (2008). Rastrojos y algo más. Historia de la siembra directa en Argentina. Buenos Aires: Teseo/Universidad de Belgrano.

Angenot, M. (2010). El discurso social. Los límites de lo pensable y lo decible. Buenos Aires: Siglo XXI.

Balsa, J. (2007). Las disputas hegemónicas en torno de las cuestiones sociales agrarias de la pampa argentina en la actualidad. En Girbal-Blacha, N. yMendonça, S. (coords.). Cuestiones agrarias en Argentina y Brasil(pp. 149-170). Buenos Aires: Prometeo.

Balsa, J. (2012). Formaciones discursivas y disputas por la hegemonía en torno a los modelos de desarrollo agrario. En Lázzaro, S. y Balsa, J. (Coords.). Agro y política en Argentina. El modelo agrario en cuestión, 1930-1943 (pp. 35-117). Buenos Aires: CICCUS.

Balsa, J. (2013). Los avatares de la reforma de la ley de colonización durante el primer peronismo (1946- 
1955). En Girbal-Blacha, N. y Mendonça, S. (Dirs.). Corporaciones agrarias y políticas públicas en América Latina (pp. 193-226). Rosario: Prohistoria.

Bocchicchio, A. y Cattáneo, C. (2005). Transformaciones en la agricultura e innovación organizacional en asociaciones de productores: los casos de AAPRESID y ASAGIR. En Benencia, R. y Flood, C. (Comps.). Trayectorias y contextos. Organizaciones rurales en la Argentina de los noventa (pp. 89-104). Buenos Aires: La Colmena.

Carini, G. (2014a). Reivindicaciones, discursos y estrategias en mutación: la Sociedad Rural de Río Cuarto en el marco de una 'nueva agricultura' (1990-2000). En Olivera, G. (Dir.). El agro cordobés en el siglo XX: entramados productivos, políticos y sociales desde una perspectiva histórica (pp. 223-280). Córdoba: Editorial de la Secretaria de Investigación, Ciencia y Técnica de la Facultad de Filosofía y Humanidades. Universidad Nacional de Córdoba.

Carini, G. (2014). Reivindicaciones, discursos y conflicto: las corporaciones agrarias en el marco de la estructuración del ‘consenso' neoliberal (1989-1991). Conflicto Social 7 (12), pp. 88-113. Recuperado de: http://publicaciones.sociales.uba.ar/index.php/CS/article/view/579

Carini, G. (2015). Nuevo Estado, viejos intereses: corporaciones agrarias y mediación política en el interior de Córdoba (1935-1955). Estudios del ISHIR. 5 (11), pp. 93-113. Recuperado de: http://web2.rosarioconicet.gov.ar/ojs/index.php/revistaISHIR/article/view/421

Carini, G. (2016). Debates y controversias en el marco de la normalización de las prácticas historiográficas: Jorge Sábato y la formación de la clase dominante en Argentina. En Basconzuelo, C. y Maldonado, G. (Comps.). Construyendo investigadores en ciencias sociales (pp. 303-321). Río Cuarto: UNIRÍO.

Carini, Gabriel Fernando (2017). “Agro, negocio y nueva institucionalidad en las pampas: itinerarios y propuestas de abordajes para el análisis de la representación de intereses agrarios”. En: Banzato, G.;Blanco, G.; Perren, J. (comps.). La Expansión de la frontera productiva y estructura agropecuaria. Buenos Aires:Prometeo-AAHE [En prensa].

Carniglia E. (2004). La mediatización de la ruralidad. Representaciones del espacio en la prensa especializada nacional. En Ciamdevilla G. y Carniglia, E. (Comps). Comunicación, ruralidad y desarrollo (pp. 285-306). Buenos Aires: Ediciones INTA.

Carniglia, E. (2011). Las ruralidades de la prensa. Agronegocio, tecnología y agrarismo. Río Cuarto: Universidad Nacional de Río Cuarto.

Carreras Doallo, X. (2012). Los medios gráficos como fuente. Un análisis de la revista La Chacra durante el peronismo clásico. Estudios Rurales, 2 (2), pp. 184-201. Recuperado de: http://ppct.caicyt.gov.ar/index.php/estudios-rurales/article/view/1394

Da Orden, M. y Melon Pirro, J. (Comps.) (2007). Prensa y peronismo. Discursos, prácticas, empresas, 19431958. Rosario: Prohistoria.

De Arce, A. (2009). Mujeres, familia y trabajo. Chacra, caña y algodón en la Argentina (1930-1960). Bernal: Universidad Nacional de Quilmes.

De Arce, A. (2011). Impresiones de la conflictividad agraria. Tres miradas sobre el agro argentino de los años treinta. En Cerdá, J. y Leite, L. (Comps.). Conflictividad en el agro argentino. Ambiente, sociedad y Estado (pp. 71-102). Buenos Aires: CICCUS.

Flood, C. (2005). Trayectorias institucionales comparadas de innovación tecnológica en la región pampeana. En Benencia, R. y Flood, C. (comps.). Trayectorias y contextos. Organizaciones rurales en la Argentina de 
los noventa (p. 137-177). Buenos Aires: La Colmena.

Eujanian, A. (1999) Historia de revistas argentinas. 1900/1950. La Conquista del público. Buenos Aires: Asociación Argentina de Editores.

Girbal-Blacha N. y Quatrochi D. (1999). Cuando opinar es actual. Revistas argentinas del siglo XX. Buenos Aires: Academia Nacional de Historia.

Gutiérrez, T. (2002). El peronismo y el "Mundo agrario”. Una visión sobre el agro argentino, 1949-1955. $\begin{array}{llllll}\text { Mundo } & \text { Agrario. } & 2 & \text { (4), } & \text { pp. } & \text { 1-14. }\end{array}$ http://www.mundoagrario.unlp.edu.ar/article/viewFile/v02n04a04/1527

Gutiérrez, T. (2004). Las representaciones del agro argentino en el peronismo y el posperonismo. La revista Mundo agrario, 1949-19621. Anuario del CEH, 4 (4), pp. 37-53.

Gutiérrez, T. (2005). Revista La Chacra: industria editorial, agro y representación (1930-1955). En Lázzaro, S. y Galafassi, G. (Comps.). Sujetos, políticas y representaciones del mundo rural. Argentina 1930-1975 (pp. 19-61). Buenos Aires: Siglo XXI.

Gras, C. (2009a). El nuevo empresariado agrario: sobre la construcción y los dilemas de sus organizaciones. En Gras, C. y Hernández, V. (Coords.). La Argentina rural. De la agricultura familiar a los agronegocios(pp. 215-237). Buenos Aires: Biblos.

Gras, C. y Hernández, V. (2013). Los pilares del modelo agribusiness y sus estilos empresariales. En Gras, C. y Hernández, V. (Coords.). El agro como negocio. Producción, sociedad y territorios en la globalización (pp. 17-49). Buenos Aires: Biblios.

Gras, C. y Hernández, V. (2013a). Asociatividad del empresariado agrícola en Argentina. AACREA y AAPRESID en perspectiva. En Muzlera, J. y Salomón, A. (Coords.). Sujetos sociales del agro argentino. Configuraciones históricas y procesos de cambio (pp. 36-67). Rosario: Prohistoria.

Hernández, V. (2009). La ruralidad globalizada y el paradigma de los agronegocios. En Gras, C. y Hernández, V. (Coords.). La Argentina rural. De la agricultura familiar a los agronegocios(pp. 39-65). Buenos Aires: Biblos.

Lattuada, M. (2003). Transformaciones institucionales en las corporaciones agrarias a fines del siglo XX. El caso de la Federación Agraria Argentina. En Bendini, M. et al (Comps). El campo en la sociología actual: una perspectiva latinoamericana (pp. 177-213). Buenos Aires: La Colmena.

Lattuada, M. (2006). Acción colectiva y corporaciones agrarias en la Argentina. Transformaciones institucionales a fines del siglo XX. Bernal: Universidad Nacional de Quilmes.

Lida, M. (2012). La rotativa de Dios. Prensa católica y sociedad. El Pueblo, 1900-1960. Biblos: Buenos Aires.

Lobato, M. (2009). La prensa obrera. Buenos Aires: Edhasa.

Muzlera, J. (2009). Chacareros del siglo XXI. Herencia, familia y trabajo en la pampa gringa. Buenos Aires: Imago Mundi.

Poggi, M. (2011a). Discursos, representaciones y debates sobre cuestiones agrarias en la prensa argentina. La “reforma agraria” en La Nación, La Opinión Anales y La Tierra - 1973. (Tesis de Maestría en Ciencias Sociales y Humanidades - Orientación en Comunicación - de la Universidad Nacional de Quilmes).

Poggi, M. (2011b). Problemática agraria y prensa escrita en la argentina de los años 70. Representaciones y debates sobre la propiedad de la tierra. (Tesis doctoral en Ciencias Sociales y Humanas de la Universidad 
Nacional de Quilmes).

Saítta S. (1998). Regueros de tinta. El diario Crítica en la década de 1920. Buenos Aires: Sudamericana.

Sidicaro, R. (1993). La política mirada desde arriba. Las ideas del diario La Nación (1909-1989). Buenos Aires: Sudamericana. 